\title{
RET Protein Expression in Colorectal Cancer; An Immunohistochemical Assessment
}

\author{
Maryam Ashkboos, Mehdi Nikbakht, Giti Zarinfard, Mitra Soleimani*
}

\begin{abstract}
Background: RET (rearranged during transfection) is a transmembrane receptor tyrosine kinase and a receptor for the GDNF-family ligands. It plays the role of a tumor suppressor in colorectal cancer. Therefore, it is expected that RET gene becomes downregulated in colorectal cancer (CRC). In this study, we evaluated immuno-histochemical expression of RET in $C R C$ and assessed its correlation with some of the clinicopathological features to study the prognostic value in CRC. Materials and Methods: In total, 60 cases of colorectal cancer (CRC) from the patients who underwent surgical gastroenterology operations were randomly selected. The samples included one tumor-rich section per case and one adjacent tumor-free section as the normal control for that case. Then, immunohistochemistry (ICH) was performed for RET on all the samples and the expression of RET was analyzed. Furthermore, the correlation of RET with clinicopathological features including age, gender, location of the tumor, grade, and stage was evaluated. Results: The expression of RET caused significant downregulation in cancer samples compared to the normal control ones $(\mathrm{P}=0.002)$. This downregulation increased in correlation to both grade and metastasis to lymph nodes $(\mathrm{P}=0.03$ $\& 0.02$ respectively). However, no correlation was found between the expression of RET and gender as well as location of the tumor. Conclusion: RET may be considered as a protein marker in CRC detection and prognosis.
\end{abstract}

Keywords: CRC- MBD1- clinicopathological features- ICH

Asian Pac J Cancer Prev, 22 (4), 1019-1023

\section{Introduction}

Colorectal cancer $(\mathrm{CRC})$ is the third leading cause of cancer-related death worldwide. One in twenty has the risk of developing CRC during their lifetime (Bardhan et al., 2013). CRC is characterized by certain genetic and epigenetic changes that induce proliferative activity and inhibit apoptosis (Fennell et al., 2018). Activating proto-oncogenes or de-activating some tumor suppressor genes can lead to cancer (Munteanu et al., 2015). DNA hypermethylation is frequently associated with transcriptional silencing of tumor suppressor genes $(\mathrm{Ng}$ and $\mathrm{Yu}, 2015)$. Several tumor suppressor genes have been already introduced that are involved in the CRC pathogenesis. Among the most widely known genes are $A P C, p$ 53, p27, MSI, LOH 18q, deletion $5 q$ allele, and DNA hypermethylation (Munteanu et al., 2015). DNA hypermethylation is a type of aberrant epigenetic alteration. CRC alterations were first identified in 1980s (Armaghany et al., 2015). A subtype of CRC, i.e. CIMP ( $\mathrm{CpG}$ island methylator phenotype) is characterized as an epigenome fraught with methylated genes. CIMP CRCs are associated with older age, female gender, family history of CRC, proximal location in the colon, and mucinous cell differentiation (Weisenberger et al.,
2006). Recently, it is found that RET (rearranged during transfection) plays a tumor suppressor role in CRC (Luo et al., 2013). RET is a transmembrane receptor tyrosine kinase and a receptor for the GDNF-family ligands. It was one of the first oncogenes that was identified. It has long been reported that specific mutations in RET are associated with medullary thyroid carcinoma (Mulligan et al., 1994). The association between RET and hirschsprung's disease (developmental disorder of the enteric nervous system) is well-recognized as its major responsible gene (Tomuschat and Puri, 2015). Recently, the tumor suppressor functions of RET have been taken into consideration. It has been shown that RET is a conditional tumor suppressor gene in the CRC through its dual role: mutation and aberrant methylation (Grady, 2013). In a recent study, it was concluded that RET is the second of nine genes that exclusively hypermethylate in CIMP CRC (Wei et al., 2016). Considering the tumor suppressor functions of RET in CRC and since in CIMP, RET expression is correlated to clinicopathologic features, in this study, we aim to assess the RET expression in cancer and normal tissues and to evaluate the correlation of RET expression with clinicopathological features.

Department of Anatomical Sciences, Isfahan University of Medical Sciences, Isfahan, Iran. *For Correspondence: mitrasoleimani1@gmail.com 


\section{Materials and Methods}

\section{Case Selection and Tissue Samples}

In total, 60 cases of colorectal cancer (CRC) and 60 cases of normal adjacent tissues (more than $10 \mathrm{~cm}$ far from the margin of tumors) were collected as paraffin embedded blocks prepared at Alzahra Hospital (Isfahan, Iran) and assessed in a longitudinal retrospective study. All the patients sustained either elective or emergency surgical operations. The non-resected tumors or those with antineoplastic therapies were excluded from the study. The frequency and distribution of clinicopathologic features are depicted in Table 1.

\section{Immunohistochemistry (ICH)}

Immunohistochemical analysis of RET was performed as previously described protocol (Yemelyanova et al., 2011). Briefly, the sections with $5 \mu \mathrm{m}$ thickness from formalin-fixed paraffin-embedded blocks were deparaffinized in xylene and rehydrated in a graded series of ethanol. Next, the sections pretreated with $0.01 \mathrm{~mol} / \mathrm{L}$ citrate buffered saline $(\mathrm{pH} 6.0)$ and autoclaved at $121^{\circ} \mathrm{C}$ for $15 \mathrm{~min}$ for antigen retrieval. Endogenous peroxidase activity was blocked by incubation with $3 \% \mathrm{H}_{2} \mathrm{O}_{2}$ for $30 \mathrm{~min}$ at the room temperature. Nonspecific binding of the immunological reagents was blocked by incubating the sections with $10 \%$ normal goat serum for $1 \mathrm{~h}$. Then, the sections were incubated with rabbit antibody against human RET (\#ab134100, Abcam, UK) at $4^{\circ} \mathrm{C}$ overnight and (after washing) incubated with anti-rabbit secondary antibody (\#ab205718) for $30 \mathrm{~min}$ RT. The peroxidase activity was developed with 3,3'-diaminobenzidine etrahydrochloride (DAB) in the sterile $\mathrm{H}_{2} \mathrm{O}_{2}$ solution for 5 min. Nuclear counterstaining was done using Mayer's hematoxylin solution. Two blinded observers (Sh.G and
ZA) independently examined the immunostained sections.

\section{Cell counts and scoring system}

Stained sections were observed by the light microscope and at least 500 cells in 10 different fields were picturized by Motic Image Advanced Plus 3. Cell count was done in Image J $1.52 \mathrm{n}$ software. The staining intensity was assessed on a four-point scale as follows: $0=$ Negative, $1=$ Weak, $2=$ Intermediate, and 3= Strong (Figures 1B and $2 \mathrm{~B})$. Total intensity per section was calculated by the following equation: $\mathrm{H}$-score $=\Sigma(1+\mathrm{i})$ pi.

\section{Statistical analysis}

Statistical analysis was performed in SPSS software (v. 25) using paired t-student test, independent t-test, Pearson's correlation coefficient, and Spearman's rank correlation coefficient to compare protein expression difference between the tumor and normal adjacent samples and to evaluate the correlation of RET expression with the features of gender, age, location, type, grade, and stage of tumor. Data were represented as mean $\pm \mathrm{SD}$ and p-values of less than 0.05 were considered as statistically significant. $\mathrm{P}<0.05$ was considered significant.

\section{Results}

\section{General observation of samples}

The cells with brown stained cytoplasm for RET with various staining in non-mucinous (Figures $1 \mathrm{C}$ and $\mathrm{D}$ ) and

Table 1. The Mean Expression of RET Protein in Cancer Samples Compared to Normal Adjacent Samples

\begin{tabular}{lccc}
\hline & Mean & SD & P-value \\
\hline Cancer & 50.9 & 7.8 & $<0.002$ \\
Normal & 107.01 & 14.7 & \\
\hline
\end{tabular}

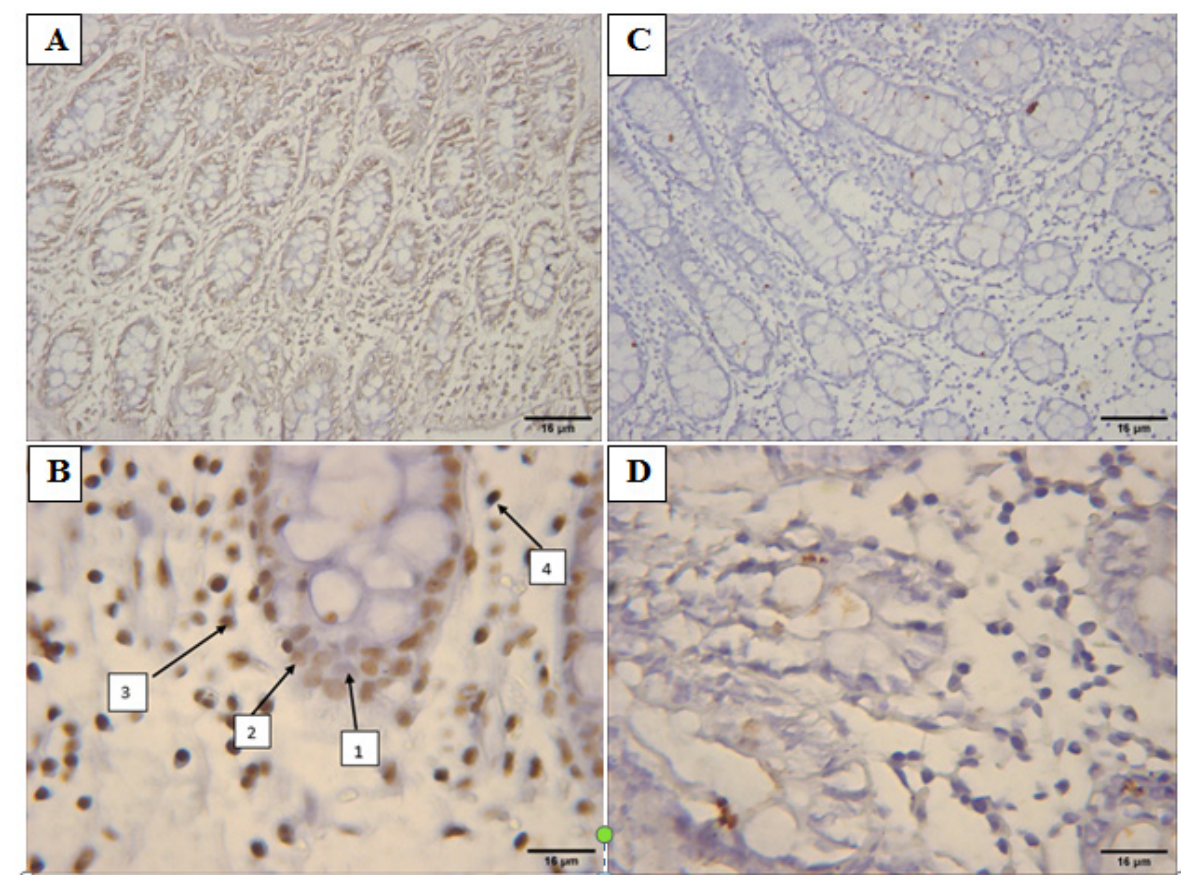

Figure 1. A \& B, The Expression of RET in Normal Sections Compared to C \& D, CRC(Non-Mucinous) Stained Samples (100 \& 400X Magnification). Brown stained cells are considered as positive cells for RET. B) Different intensity of positive stained cells for $\operatorname{RET}(1=$ non-stained, $2=$ mild, $3=$ moderate, $4=$ sever $)$ 


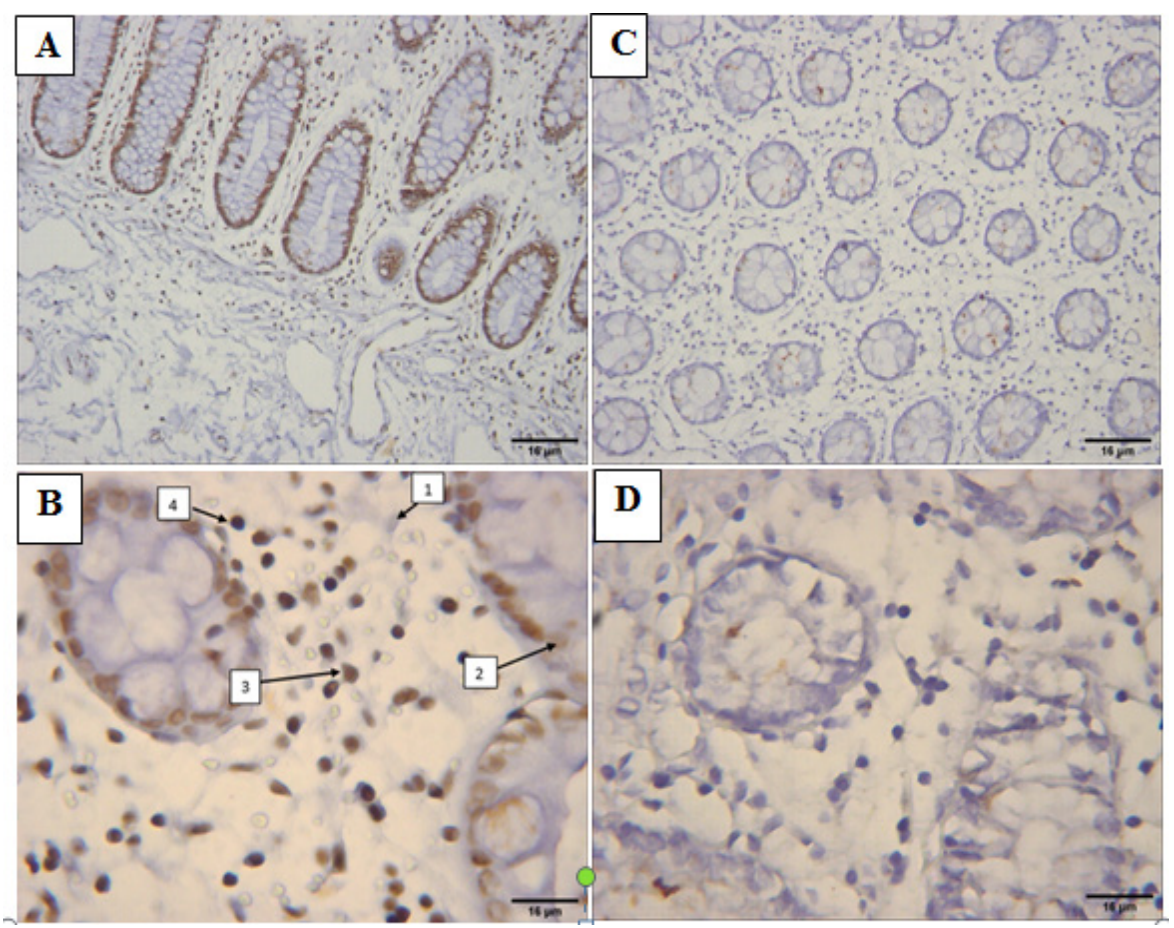

Figure 2. A \& B, The Expression of RET in Normal Sections Compared to C \& D, CRC (Mucinous) Stained Samples (100 \& 400X Magnification). Brown stained cells are considered as positive cells for RET. B) Different intensity of positive stained cells for $R E T$ ( $1=$ non-stained, $2=$ mild, $3=$ moderate, $4=$ sever $)$

mucinous (Figures $2 \mathrm{C}$ and $\mathrm{D})$ cancer sections and normal sections (Figures 1 (A and B) and Figures 2 (A and B) were observed. Significant reduction in RET protein expression was noted in the tumor samples in comparison to the normal control samples.

Table 2. The Mean Expression of RET Protein in Cancer Samples Compared to Normal Adjacent Samples.

\begin{tabular}{lccccc}
\hline & \multicolumn{2}{c}{ Male } & \multicolumn{2}{c}{ Female } & P-value \\
& Mean & SD & Mean & SD & \\
\hline Cancer & 47.5 & 12.6 & 53.1 & 9.9 & 0.21 \\
Normal & 140.4 & 25.1 & 89.03 & 17.7 & 0.046 \\
\hline
\end{tabular}

Correlation of RET expression and clinicopathologic features

The statistical analysis of the data showed a significant difference in the expression of RET in cancer samples in comparison to the normal adjacent samples $(\mathrm{P}<0.002)$. No significant correlation was found between the expression

Table 3. The Expression of RET Protein Expression Correlation to Gender

\begin{tabular}{lcc}
\hline & \multicolumn{2}{c}{ Age } \\
& $\mathrm{r}$ & P-value \\
\hline Cancer & -0.031 & 0.81 \\
Normal & -0.275 & 0.03 \\
\hline
\end{tabular}

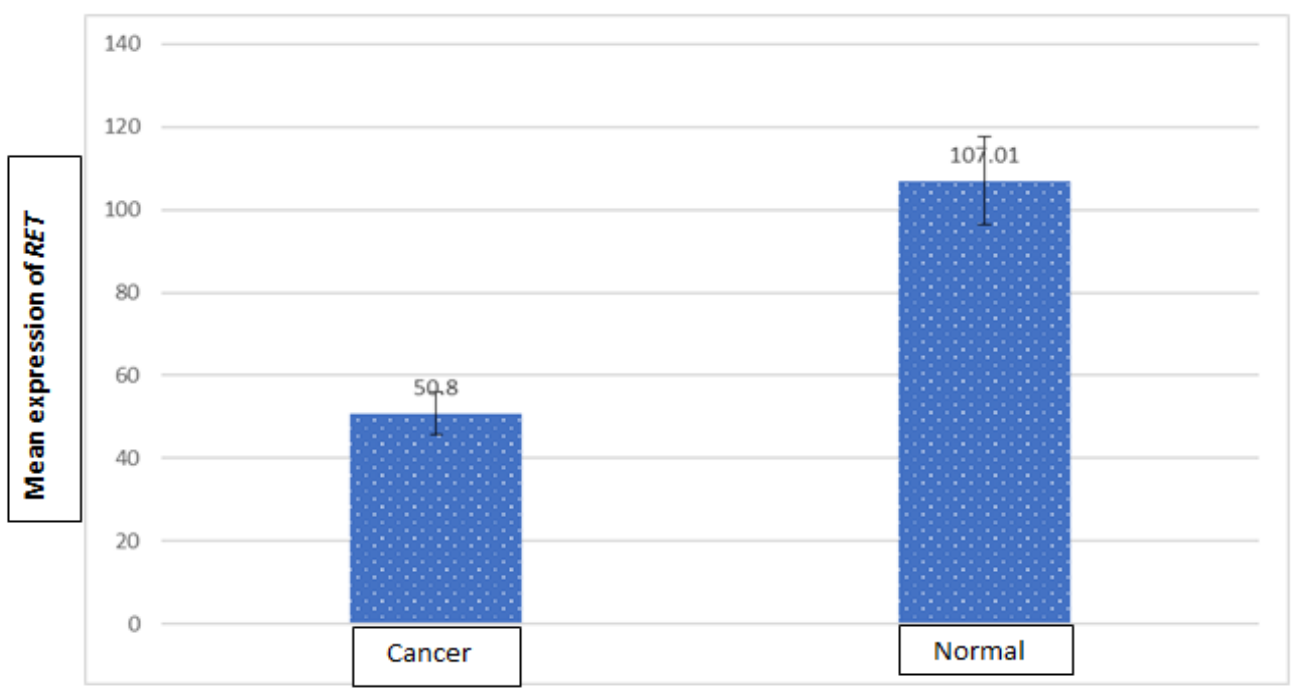

Figure 3. The Mean Expression of RET Protein in Cancer Samples Compared to Normal Samples 
Table 4. The Pearson Correlation Coefficient of RET Protein Expression Correlation to Age

\begin{tabular}{lccc}
\hline Tumor Type & Mean & SD & P-value \\
\hline Mucinous & 53.1 & 8.5 & 0.53 \\
Non-mucinous & 39.8 & 20.1 & \\
\hline
\end{tabular}

Table 5. The Mean Expression of RET Protein in Cancer Samples Correlation to Tumor Type

\begin{tabular}{lccc}
\hline Tumor Location & Mean & SD & P-value \\
\hline Colon & 48.4 & 9.8 & 0.64 \\
Rectosigmoid & 56.3 & 13.2 & \\
\hline
\end{tabular}

Table 6. The Mean Expression of RET Protein Correlation to Tumor Location

\begin{tabular}{lccc}
\hline Lymph node metastasis & Mean & SD & P-value \\
\hline Yes & 53.3 & 8.1 & 0.02 \\
No & 4.9 & 1.7 & \\
\hline
\end{tabular}

Figure 4. The Mean Expression of RET Protein in Cancer Samples Compared to Normal Samples Correlation to Tumor Type

\begin{tabular}{lcc}
\hline & \multicolumn{2}{c}{ RET Expression } \\
& $\mathrm{r}$ & P-value \\
\hline Stage & -0.157 & 0.28 \\
Grade & -0.248 & 0.03 \\
\hline
\end{tabular}

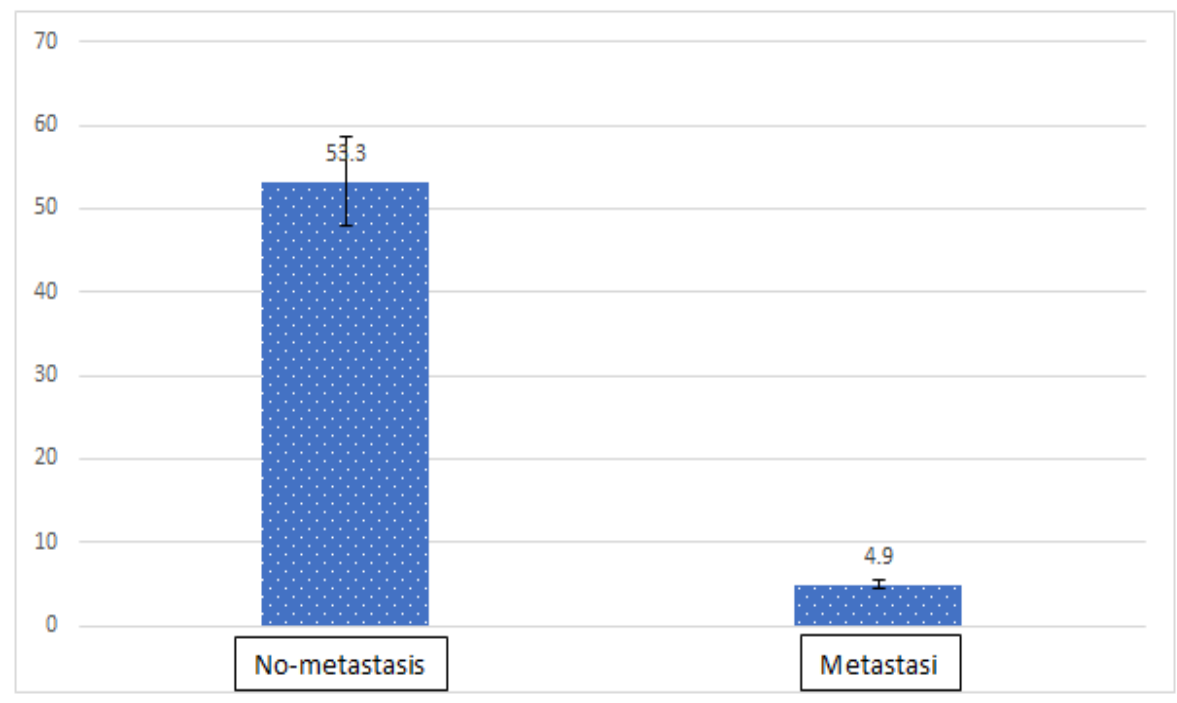

Figure 4. The Mean Expression of RET Protein in Cancer Samples Compared to Normal Samples Correlation to Tumor Type

of RET and gender $(\mathrm{P}=0.21)$. However, the expression of $R E T$ was significantly higher in the normal female samples compared to the normal male samples $(\mathrm{P}=0.046)$. Pearson's correlation coefficient showed no significant correlation between RET expression and age $(\mathrm{P}=0.54)$. Although a conversely significant correlation was seen between RET expression and age in the normal samples $(\mathrm{P}=0.03)$, there was no significant correlation between $R E T$ expression in cancer samples compared to the normal samples in terms of the type and location of the tumors $(\mathrm{P}=0.53$ and 0.64 , respectively). Moreover, a significant difference was found in RET expression between stages $(\mathrm{P}=0.28)$. However, $R E T$ expression showed a significant difference in the samples with metastasis to lymph nodes from the samples without metastasis to lymph nodes (0.02). Spearman's rank correlation coefficient showed a significant difference in RET expression in terms of the grade of tumors $(\mathrm{P}=0.03)$. The statistics is described in Tables 3-7 and Figures 3-4.

\section{Discussion}

In this study, we assessed the expression of RET in colorectal cancer and evaluated the correlation of $R E T$ expression to clinicopathologic features. RET gene is one of the first oncogenes that has been identified (Mulligan et al., 1994). Its role as a proto-oncogene has been known for many years (Nakamura et al., 1994). However, its account as a tumor suppressor gene recently is taken into consideration. It has been recently reported that RET is methylated in $63 \%$ of colorectal cancers and that, in $27 \%$ of colon adenomas, RET is in the methylated format. Therefore, tumor suppressor activity of RET in the colon cancer is suggested (Luo et al., 2013). In the integrated analysis of genome-wide DNA methylation and gene expression profiling study, RET was the second of nine potential biomarkers that was introduced for the rectal cancer (Wei et al., 2016). In our study, we found RET expression was significantly lower in CRC than in the normal samples. This was in concordance with previous studies, demonstrating the significant downregulation of RET during colon and rectal cancers. The data of this study showed that the expression of RET was in correlation with grade and metastasis to lymph nodes. However, no correlation was found between RET expression and gender, age, location, and stage of the tumors.

In conclusion, the results from this study suggested that, firstly, RET is involved in the pathogenesis of colorectal cancer. Secondly, it is in correlation with the tumor grade and metastasis. However, to discuss RET 
consideration as a prognosis factor with more certainty, a larger sample size than the one used in this study is required.

\section{Author Contribution Statement}

The authors confirm contribution to the paper as follows: study conception and design: Mitra Soleimani, Maryam Ashkboos, Mehdi Nikbakht; data collection: Mehdi Nikbakht, Maryam Ashkboos; analysis and interpretation of results: Mitra Soleimani, Maryam Ashkboos, Giti Zarinfard; draft manuscript preparation: Mitra Soleimani. All authors reviewed the results and approved the final version of the manuscript.

\section{Acknowledgements}

This study was supported by Vice-Chancellery for Research and Technology, Isfahan University of Medical Sciences under grant no. 397385.

\section{Conflict of interest}

The authors declare no conflict of interest.

\section{References}

Armaghany T, Wilson JD, Chu Q, Mills G (2012). Genetic alterations in colorectal cancer. Gastrointestinal Cancer Res, 5, 19.

Bardhan K, Liu K (2013). Epigenetics and colorectal cancer pathogenesis. Cancers, 5, 676-713.

Fennell L, Dumenil T, Hartel G, et al (2018). Genome scale epigenetic profiling reveals five distinct subtypes of colorectal cancer. Bio Rxiv, 2018, 397620.

Grady WM (2013). Context is everything for dependence receptors in colorectal cancer. Proc Nat Acad Sci U S A, 110, 2697-8.

Luo Y, Tsuchiya KD, Park DI, F, et al (2013). RET is a potential tumor suppressor gene in colorectal cancer. Oncogene, 32, 2037.

Mulligan LM, Eng C, Healey CS, et al (1994). Specific mutations of the RET proto-oncogene are related to disease phenotype in MEN 2A and FMTC. Nat Genet, 6, 70.

Munteanu I, Mastalier B, Munteanu Ml, et al (2015). The relationship between K-Ras gene mutation, some degree of differentiation and tumor markers in advanced colorectal cancer. Manage Health, 18.

Nakamura T, Ishizaka Y, Nagao M, Hara M, Ishikawa T (1994). Expression of the ret proto-oncogene product in human normal and neoplastic tissues of neural crest origin. J Pathol, 172, 255-60.

$\mathrm{Ng} \mathrm{J,} \mathrm{Yu} \mathrm{J} \mathrm{(2015).} \mathrm{Promoter} \mathrm{hypermethylation} \mathrm{of} \mathrm{tumour}$ suppressor genes as potential biomarkers in colorectal cancer. Int J Mol Sci, 16, 2472-96.

Tomuschat C, Puri P (2015). RET gene is a major risk factor for Hirschsprung's disease: a meta-analysis. Pediat Surg Int, 31, 701-10.

Wei J, Li G, Zhang J, et al (2016). Integrated analysis of genome-wide DNA methylation and gene expression profiles identifies potential novel biomarkers of rectal cancer. Oncotarget, 7, 62547.

Weisenberger DJ, Siegmund KD, Campan M, et al (2006). $\mathrm{CpG}$ island methylator phenotype underlies sporadic microsatellite instability and is tightly associated with BRAF mutation in colorectal cancer. Nat Genet, 38, 787.

Yemelyanova A, Vang R, Kshirsagar M, et al (2011). Immunohistochemical staining patterns of p53 can serve as a surrogate marker for TP53 mutations in ovarian carcinoma: an immunohistochemical and nucleotide sequencing analysis. Modern Pathol, 24, 1248.

This work is licensed under a Creative Commons AttributionNon Commercial 4.0 International License. 